Bei der Ableitung der Werthe für Wasserstoff, Kohlenoxyd und Methan aus den Daten einer gemeinsamen Verbrennung findet eine theilweise Compensation der Abweichungen von der Avogadro'schen Regel statt, und darauf ist es wohl zurückzuführen, dass die für reine kohlenstoffhaltige Gase nicht unbeträchtliche Fehlerquelle bisher der Aufmerksamkeit entgangen ist.

Als genaue Gleichung ergiebt sich aus den oben abgeleiteten Grundgleichungen:

$$
\begin{aligned}
& \mathrm{H}=1.0005 \mathrm{~K}-1.00170-0.0060 \mathrm{CO}_{2} \\
& \mathrm{CO}=0.3329 \mathrm{~K}-\quad 0-1.3394 \mathrm{CO}_{2} \\
& \mathrm{CH}_{4}=0.3336 \mathrm{~K}+1.00200-0.3340 \mathrm{CO}_{2} \text {. }
\end{aligned}
$$

Für Leuchtgas mittlerer Zusammensetzung werden durch Anwendung der üblichen Formeln die Prozentzahlen für Wasserstoff, Koblenoxyd und Metban um etwa $0.25,0.2$ und 0.1 geändert. Um aber die Rechnung unter Beibehaltung der gewöhnten Form der Gleichungen im wesentlichen genau auszuführen, braucht man nur den Werth für Wasserstoff um $0.006 \mathrm{CO}_{2}$ zu vermindern, und den Werth für Kohlenoxyd um den gleichen Betrag zu erhöhen, man hat dann also:

$$
\begin{aligned}
& \mathrm{H}=\mathrm{K}-\mathrm{O}-0.006 \mathrm{CO}_{2} \\
& \mathrm{CO}=1 / 3 \mathrm{~K}-\mathrm{O}+3_{3} / 4 \mathrm{CO}_{2}+0.006 \mathrm{CO}_{2} \\
& \mathrm{CH}_{4}=-1 / 3 \mathrm{~K}+0-1 / 3 \mathrm{CO}_{2} .
\end{aligned}
$$

Die Zulässigkeit dieser einfachen Formeln ergiebt sich aus dem Vergleich mit den oben angeführten genauen Gleichungen.

Die experimentellen Versuche, auf die im Vorstehenden Bezug genommen ist, sind von Hrn. cand. chem. Eickmann bearbeitet worden.

70. A. Woh1: Vollständige Gasanalyse mittels Druckmessungen.

(7. Mittheilung über gasometrische Bestimmungen in Gaskolben 1).):

[Aus dem I. Berliner Universităts-Laboratorium.]

(Eingegangen am 28. Januar 1904.)

Das allgemeine Verfahren der rollständigen 'Gasanalyse beruht bekanntlich darauf, nacheinander Kohlensäure, schwere Kohlenwasserstoffe, Sauerstoff und Kohlenoxyd durch Absorption zu bestimmen und danach Wasserstoff und Methan im Gasreste durch fractionirte Verbrennung. Die Menge der einzelnen Bestandtheile ergiebt sich aus der

1) Vergl. diese Berichte 35, 3485, 3493 [1902; 36, 674, 676, 1412, 1417 [1903]. 
jedesmaligen Volumenverminderung; werden, wie üblich, $100 \mathrm{ccm}$ Gas bei Lufttemperatur und dem gerade herrschenden Druck zur Analys angewandt, so liefert die Differenz der Volumina, gemessen bei gleicher Temperatur und gleichem Druck, direct Procente bezw. Zahlen, die dazu in einfacher Beziehung stehen.

An Stelle der am wenigsten befriedigenden absorptiometrischen Bestimmung, der des Koblenoxyds, kann mit Vortheil - inslesondere unter Benutzung der Drehschmidt'schen, von Cl. Winkler verbesserten Platincapillare - auch die Verbrennung des Koblenoxyds zusammen mit Wasserstoff und Methan treten; dabei liefern dann Contraction, Kohlensäuregehalt und Sauerstoffserbrauch im verbrannten Gasgemenge die Bestimmungsstücke, aus denen sich die drei Bestandtheile rechnungsmässig ergeben.

Die nachstehend beschriebene Methode schliesst sich in allen Punkten der letzteren, wohlbekannten gasanalytischen Arbeitsweise an, nur dass Gasbüretten und Gaspipetten durch Gaskolben und die Volumenmessungen durch Druckbestimmungen ersetzt sind. Auch hier wird am besten die Gasmenge so gewählt, dass die unmittelbar beobachteten Druckdifferenzen Werthe ergeben, die zu den Procentzahlen in einfacher Beziehung steben. Ein bequemes Verfahren, daza von vornherejn bestimmte, nach ihrem Partialdruck bemessene Gasmengen in Gaskolben einzuführen, sei zunächst beschrieben.

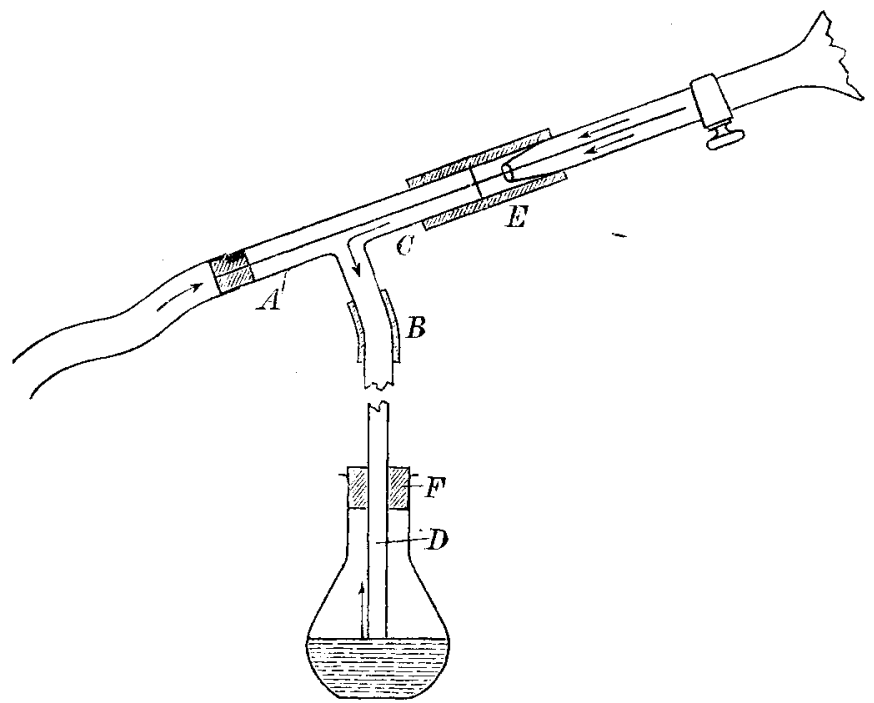

Fig. 1. 
Die Gaszuführung erfolgt darch eine kurze Metallcapillare, die mittels Gummistopfen oder Einsiegeln in einem T-Stück $A B C$ befestigt ist (Fig. 1); an dem abwärts gerichteten Ende $B$ des $T$. Stücks ist mittels Gummischlauch ein etwas längeres Glasrohr $D$ angebracht, das durch einen Wasserverschluss abgeschlossen wird. Die Metallcapillare, die 4-5 cm über das Ende des T-Stücks $C$ herausragt, wird in den Hahnansatz des Gaskolbens hineingesteckt und Hahnansatz und T.Stück durch Gummischlauch $E$ mit einander verbunden. Alsdann erfüllt ein durch die Metallcapillare eingeleiteter Gasstrom durch Verdrängen den Hahnansatz, das T.Stück und das angesetzte Glasrohr mit dem zu untersuchenden Gase, und dieses strömt durch den Wasserrerschluss ab. Der Gaskolben war zuror bis zu einem bestimmten Minderdruck evacuirt worden (am besten regelmässig bis $700 \mathrm{~mm}$ ). Wird nun der Kolbenhahn capillar geöffnet, so tritt der Gasstrom ganz oder theilweise in den Kolben ein. Dass der Gaskolben nabezu gefüllt ist, erkennt man daran, dass auch bei starkem Drosseln des Gaszuflusses noch Blasen aus dem Fläschschen entweichen. Alsdann schliesst man für einen Augenblick Kolbenhahn und Gasquelle $a b$, und öffnet dann wieder langsam den ersteren. Steigt das Wasser noch sebr weit zurück, so leitet man in den Gaskolben ron neuem Gas ein; im anderen Fall, wenn die Sperrflüssigkeit des Wasserverschlusses nur etwa $3-4 \mathrm{~cm}$ hoch zurücksteigt, löst man nach dem Schliessen der beiden Hähne das Indicator-Kölbchen von dem Stopfen $F$, wobei die Wassersäule im Rohr als Verschluss haften bleibt. Dann öffnet und schliesst man noch einmal für einen Augenblick den Gaszuführungshabn und unmittelbar darauf den Kolbenbahn. Auf diese Weise ist der Kolben mit Gas gefüllt bis zum Gleichgewicht mit dem äusseren Druck (bis zum Miuderdruck O), ohne dass Luft irgendwie als Verunreinigung zutreten konnte; war der Kolben vorber auf $700 \mathrm{~mm}$ evacuirt, so beträgt demnach der Partialdruck des zugelassenen Gases $700 \mathrm{~mm}$. Die praktische Ausfübrung erfordert etwa 2-3 Min.

In den früheren Abhandlungen (I. c.) war speciell nur die absorptiometrische Bestimmung von Kohlensäure und Sauerstoff erörtert worden. Um das Verfahren zu einer allgemeinen gasanalytischen Methode auszugestalten, war jetzt noch die Bestimmung der schweren Kohlenwasserstoffe und die Verbrennungsanalyse zu bearbeiten.

Die für die Bestimmung der schweren Kohlenwasserstoffe sonst vorgeschriebene rauchende Schwefelsäure ron etwa $21 \mathrm{pCt}$. Schwefelsäureanhydrid ist für die Füllung der Hempel'schen Gaspipetten wohl geeignet; für die hier erforderliche Handbabung des Absorptionsmittels, das in den Gaskolben eingesaugt und wieder abgesaugt werden muss, ist dieselbe unbequem und auch nicht ungefährlich. Versuche, die schweren Kohlenwasserstoffe zu bestimmen 
mit einem Gemenge von Salpeterschwefelsäure und Brom, scheiterten daran, dass gleichzeitig vorhandenes Kohlenoxyd sich stark oxydirt ${ }^{1}$ ). Dann aber ergab sich, dass bei der intensiven Berührung von Gas und Flüssigkeit, wie es beim Schütteln im Gaskolben herbeigefübrt wird, zur raschen und rollständigen Absorption auch schon eine rauchende Schwefelsäure von 5-7 pCt. Gebalt an Schwefelsüureanhydrid genügt, die sehr bequem zu handhaben ist und überdies die Hähne vorzüglich dicht hält.

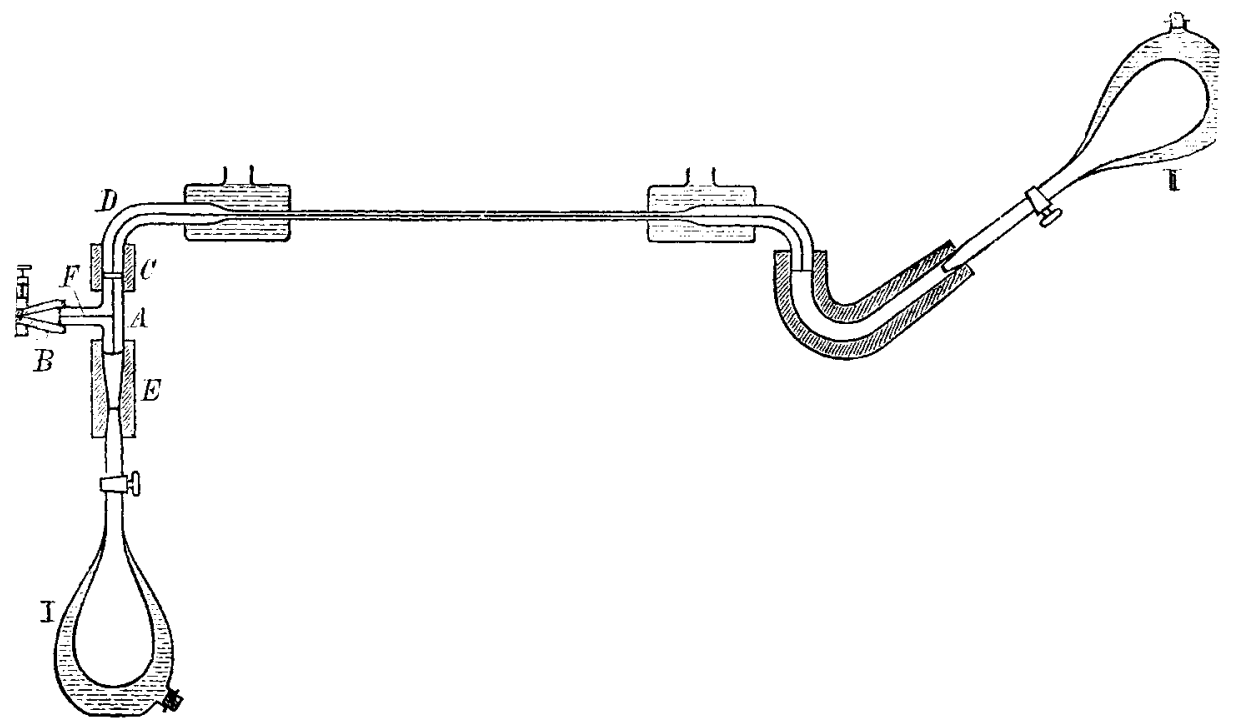

Fig. 2.

Zur Ausfübrung der Verbrennung war zunächst der Weg gewählt worden, den durch Ansaugen von Sauerstoff oder Luft verbrennlich gemachten Gasrest durch die erhitzte Platincapillare hindurch in einen zweiten evacuirten Gaskolben überzusangen, bis Druckgleichgewicht eintrat. Die Gaskolben sind dabei mit starkem Gummischlauch an die beiden Enden der Platincapillare angeschlossen und ein kleines, metallenes T-Stück (Fig. 2) ror einen der Kolben eingeschaltet, sodass der Luftinhalt der beiden Habnansätze wie der Platincapillare vor Beginn der Verbrennung durch Evacuiren mit der Wasserstrablpumpe praktisch vollständig entfernt werden kann. Mau lässt dann durch die, wie üblich, erhitzte Capillare hindurch das $G a s$ in den evacuirten Gaskolben II überströmen; der Letztere ist in schräger Lage, die Spitze nach unten, befestigt, sodass ein Wassertröpfchen, das

1) Vergl. Treadwell und Stokes, diese Berichte 21, 3131 [1888]. 
sich über der Habnbohrung ansammelt, als Indicator für den Gang der Gasblasen dienen kann. Man schliesst hinterher die verbindenden Schläuche unmittelbar vor und hinter der Platincapillare durch aufgeschobene Schraubenquetschhähne ab, saugt in beide Kolben vach Entfernung von der Platincapillare luftfreies Wasser ein, sodass der Gasinhalt von Schlauch und Hahnansätzen mit in die Kolben gelangt und führt die Druckbestimmungen aus.

Die Ausführung des Verfahrens war ganz bequem. aber die Rechnung gestaltete sich schon minder einfach, wenn beide Kolben nicht genau gleichen Inhalt haben; durch den wechselnden Widerstand der Platincapillare wurde ferner das Ueberströmen bis zum genau gleichen Druck in beiden Kolben unsicher, sodass in beiden Gaskolben Druckbestimmungen erforderlich waren, und die überströmende Gas. menge war verhältnismässig klein und stellte je nach dem Barometerstand und der Grösse der Contraction einen jedesmal besonders zu berechnenden Antheil der angewendeten Gasmenge dar.

Aus diesem Grunde war es ein wesentlicher Fortschritt der Methode, dass sich ein recht einfacher Weg fand, die gesammte Menge des mit Sauerstoff oder Luft gemengten Gasrestes unter vollständiger Verbrennung in den zweiten evacuirten Gaskolben überzuführen. Um dies zu ermöglichen, wurde der folgende $W$ eg eingeschlagen:

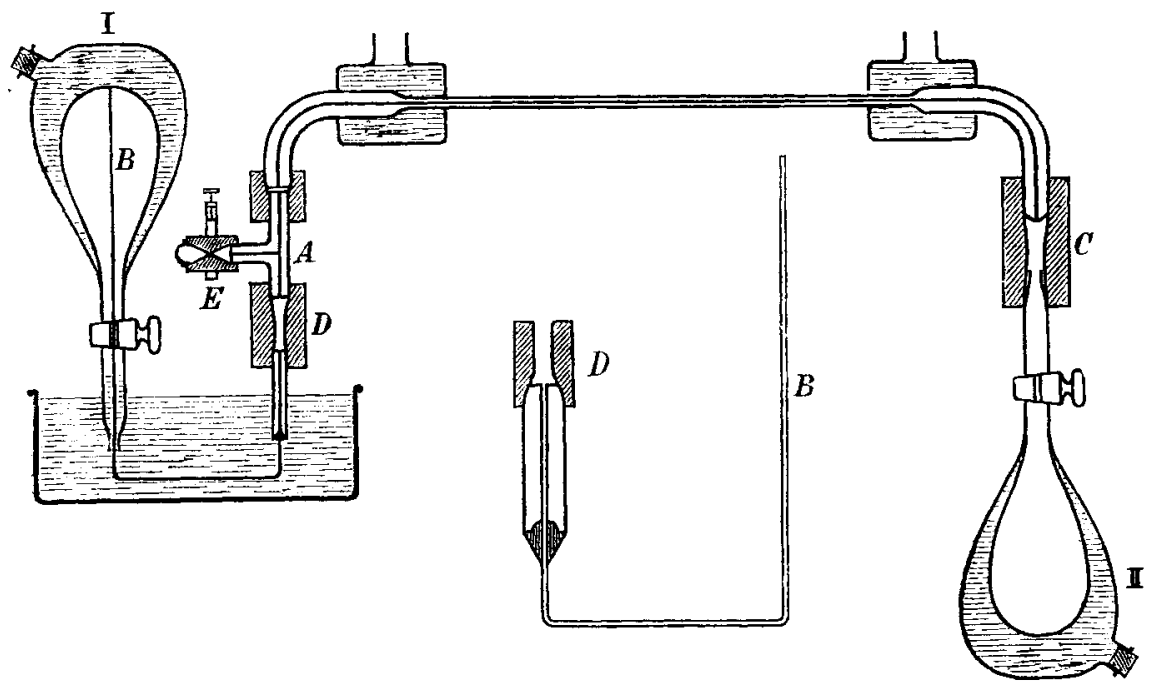

Fig. 3.

Fig. 3a.

An das nach unten gerichtete Ende des Messing.T-Stückes $A$ (Fig. 3) wurde mittels eines kurzen Stückes capillaren Druckschlauches 
eine Glascapillare (ron $4 \mathrm{~cm}$ Länge, Fig. 3a) angeschlossen, in der mit Siegellack eine Messingcapillare von $1 \mathrm{~mm}$ lichte $\mathbf{W}$ eite befestigt ist. Diese Messingcapillare ist zwei Mal umgebogen, und das nach oben gehende freie Ende $B$ besitzt eine Länge, die etwas mebr als die vollständige Höhe des Kolbens I beträgt. Es kann so dieser Kolben bei geöffnetem Ilabn, dessen Durchbohrung natïrlich exact gearbeitet sein muss, direct auf die Capillare aufgespiesst werden, wäbrend die Mündung des Hahnansatzes in ein untergestelltes, mit Wasser gefülltes Gefäss eintaucht. Wird dann durch capillares Oeffnen des Hahns an dem Kolben II das Gas unter gleichzeitiger Verbrennung übergesaugt, so steigt in den Kolben I Wasser nach und tritt nach vollständiger Verdrängung des Gasinhaltes durch die Messing- und Platin-Capillare bindurch nach Kolben II über.

Ausser diesem Fortschritt der apparativen Einrichtung und Ausführung war es nothwendig, eine ganze Reihe von Fehlerquellen bei der Verbrennung kohlenstoffhaltiger Gase aufzufinden und zu beseitigen, ehe es gelang, auf dem ersten oder zweiten oben erwähnten Wege regelmässig befriedigende Werthe zu erhalten. Die auf die Beзeitigung der Feblerquellen bezüglichen Versuche sind überwiegend nocb unter Benutzung der erstbeschriebenen apparativen Einrichtung ausgeführt worden. In den folgenden Darlegungen wird aber.der Einfachheit halber nur auf die zweite Methode des vollständigen Uebersaugens des verbrennbaren Gasgemisches Rücksicht genommen. Bezüglich der Berechnung der Ergebnisse für die erste Methode sei auf die ansführliche Beschreibung in der Dissertation des Hrn. Eickmann verwiesen.

Experimenteller Theil.

(Bearbeitet ron Hrn, cand, chem. Eickmann.)

Für die im Folgenden mitgetheilten Bestimmungen dienten je 2 wei mit Wassermantel und angeschmolzenem Hahn versehene Gaskolben von ungefähr $100 \mathrm{ccm}$ Inhalt, die als Kolben I und II bezeichnet werden. Ihr Inhalt war durch Auswägen mit Wasser von Zimmertemperatur auf Centigramm genau festgestellt (z. B. I = 10599 ; $\mathrm{II}=105.66)$; mithin betrug in diesem Falle der Correctionsfactor für das Verhältniss beider Kolben $\frac{v_{2}}{v_{1}}=\begin{gathered}1 \\ 1.008\end{gathered}$.

Zur genauen Einstellung der Temperatur im Wassermantel der Gaskolben diente ein Thermometer mit $1 / 30^{0}$-Theilung. Das bei den Analysen angewandte evacuirte Wasser wurde nach der früheren Vorschrift durch kurzes Schütteln am Vacuum hergestellt und zwar zweckmässig unter Einhaltung eines Minderdruckes, der dem zuletzt 
für den Gaskolben bestimmten Minderdruck nahe lag. Dadurch wird verhindert, dass die in den Gaskolben eingesaugte kleine Wassermenge irgendwie merklicbe Mengen der schwer löslichen Gase abgiebt oder auflöst.

Temperatureinstellungen sind vor jeder einzelnen Druckbestimmung auszuführen, und zwar unter Beibehaltung der zuerst eingestellten Temperatur bei derselben Analyse; ferner ist es nothwendig, eine Temperatur zu wählen, die der Zimmertemperatur möglicbst nahe liegt.

Die Art der Druckmessung im allgemeinen wie die Handhabung der absorptiometrischen Bestimmung von Kohlensäure und Sauerstoff im Gaskolben ist früher ausführlich beschrieben worden (diese Berichte 35 , 3493 ff. [1902]), sodass bei der folgenden Darlegung zur Vermeidung ron Wiederholungen überall darauf Bezug genommen wird.

\section{Verbrennung von reinem Wasserstoff.}

L'm den Kolben I mit Wasserstoff zu füllen, wurde derselbe zunächst an der Wasserstrahlpumpe so weit als möglich evacuirt, dann mittels der oben beschriebenen Einrichtung (Fig. 1) bis zum Gleichgewicht mit dem äusseren Druck Wasserstoff eingelassen, der in einem Kipp'scben Apparat aus Zink und Salzsäure entwickelt und je ein Mal mit Kalilauge und Wasser gewaschen war. So mit Wasserstoff gefüllt, wurde der Kolben noch ein Mal so weit als möglich evacuirt und wiederum mit Wasserstoff gefüllt. Nach Einstellung der Temperatur des Wassermantels auf 20 "wurde der Kolben auf $400 \mathrm{~mm}$ Minderdruck evacuirt. Die Wasserstoffmenge betrug dann, wenn mit $B^{\prime}$ der Barometerstand nach Abzug der Wassertension bezeichnet wird, $B^{\prime}-400 \mathrm{~mm}$. Wurde dann bis zum Gleicbgewicht mit dem äusseren Druck Sauerstoff zugelassen, so war die Sauerstoffmenge $400 \mathrm{~mm}$. Es wurde Sauerstof aus einer Bombe benutzt, dessen Gebalt ermittelt war durch absorptiometrische Untersuchung mit alkalischer Pyrogallollösung nach der früheren Vorschrift. Drei so ausgeführte Analysen lieferten übereinstimmend $89.8 \mathrm{pCt}$. O.

Da nach den üblichen gasanalytischen Methoden ein mebrmaliges Durchleiten durch die glïhenden Platincapillare vorgeschrieben ist, dies aber bei der hier in Betracht kommenden Versuchsanordnung nicht möglich wäre, sollte die Vollständigkeit der Verbrennung zunächst durch recht langsame Ausführung gesichert werden, wobei durch Stellung des Habnes ron Kolben II und Beobachtung des darüber stebenden Wassertröpfchens die Verbrennungsdauer regulirt werden konnte. Es warde mit Verbrennungszeiten begonnen, die 1/2 Stunde und mehr betrugen, aber Werthe erhalten, die um mehrere Prozente von der Theorie und untereinander abwichen. Als die Verbrennungs- 
zeit auf 5-10 Minuten herabgemindert wurde, zeigte sich wesentlich bessere Uebereinstimmung, und befriedigendere Resultate wurden erhalten, wenn der Hahn von Kolben II so gestellt wurde, dass Ueberströmen und Verbrennen des Gases in etwa 1 Minute erledigt waren.

Die Richtigkeit der einzelnen Analyse wird controllirt durch Uebereinstimmung der Werthe für Wasserstoff, berechnet ans der Contraction und aus dem Sauerstoffrerbrauch, unter einander und mit der Theorie. $\mathrm{Ob}$ die Verbrennung vollständig war, ist ausserdem daran erkennbar, dass im Kolben II nach Absorption des unverbranchten Sauerstoffs und unter Abzug des mitübergeströmten Stickstoffs der ursprüngliche Minderdruck von $700 \mathrm{~mm}$ mit genügender Annäherung wieder erreicht wird. Die so berechnete Zahl wird bei der Zusammenstellung der Analysen als »Enddruck * bezeichnet.

Im Uebrigen wird die Berechnung, wie folgt, gelührt.

Der Minderdruck im Kolben II sei $p_{1}$, der nach der Sanerstoffabsorption gemessene: p2. $_{2}$.

Aus der Gleichung:

$$
\begin{aligned}
& 2 \mathrm{H}_{2}+\mathrm{O}_{2}=2 \mathrm{H}_{2} \mathrm{O} \\
& 2.0034 \mathrm{Vol} .+1 \mathrm{Vol} .=0
\end{aligned}
$$

hat man, wenn man die Contraction mit $\mathrm{K}$ und den verbrauchten Sauerstoff mit $\mathrm{O}$ bezeichnet: $1 . \mathrm{H}=\frac{2}{3} \mathrm{~K}$ und 2. $\mathrm{H}=2.0034 \mathrm{O}$.

Man findet den Werth der Contraction $K$ in $m m$ Partialdruck aus folgender Ueberlegung: Fortgegangen aus dem Kolben $I$ ist die Gasmenge $v_{1} . B^{\prime}$. Nach dem Evacuiren von II auf $700 \mathrm{~mm}$ war in diesem noch die Gasmenge va $\left(B^{\prime}-700\right)$ vorbanden. Wäre das Gasgemiseh unverbrannt von I nach II geströmt, so müsste danach in II die Gasmenge $v_{2}\left(B^{\prime}-700\right)+v_{l}$. $B^{\prime}$ auftreten. Da nun aber die in II durch Messung wirklich gefundene Gasmenge nur $\mathrm{v}_{2}\left(\mathrm{~B}^{\prime}-\mathrm{p}_{1}\right)$ ist, so ergiebt sich die Contraction aus der Differenz beider Werthe, also wenn man alle Gasmengen auf das Volumen des Kolben I als Einheit bezieht:

$$
K=B^{\prime}-\frac{\mathrm{v}_{2}}{\mathrm{v}_{1}}\left(700-\mathrm{p}_{\mathrm{i}}\right)
$$

Der im Kolben II ahsorbirte Sauerstoff, der den unverbrauchten Rest darstellt, enthălt noch den Sauerstoff, der nach dem Evacuiren des Kolben IF auf 700 in dem Iuftrest $B^{\prime}-700$ enthalten ist; er beträgt $\left(B^{\prime}-700\right) \times 0.21$. Dieser Betrag, auf den Kolben I umgerechnet, ist der angewandten Sauerstoffmenge zuzufügen und davon der absorptiometriseh gefundene Sauerstoff $p_{2}-p_{1}$ abzuziehen. Die 80 erhaltene Differenz ist der verbrauchte Sauerstoff. Man hat also folgende Formeln:

$$
\text { Angew. } \begin{array}{rl}
H & =B-400 \\
\% & O=400 \times \alpha .
\end{array}
$$


Gefunden:

$$
\begin{gathered}
\mathrm{H}=\frac{2}{3} \cdot \mathrm{K}=\frac{2}{3}\left[\mathrm{~B}^{\prime}-\frac{\mathrm{v}_{2}}{\mathrm{v}_{i}}\left(700-\mathrm{p}_{1}\right)\right] \\
\mathrm{H}=2.0034 \times \mathrm{O}=2.0034\left\{400 . \alpha-\frac{\mathrm{v}_{2}}{\mathrm{v}_{1}}\left[\left(\mathrm{p}_{2}-\mathrm{p}_{1}\right)-\left(\mathrm{B}^{\prime}-700\right) .0 .21\right]\right\}
\end{gathered}
$$

( $\alpha$ bedeutet den Gehalt des angewendeten Sanerstoffs.)

Für die folgenden fünf Analysen ist das Verhältniss der beiden Kolben $\frac{\tau_{2}}{v_{1}}=\frac{}{1.004}$

\begin{tabular}{c|c|c|c|c|c|c|c}
\hline No. & $\mathrm{t}$ & B & $\mathrm{p}_{1}$ & $\mathrm{p}_{2}$ & $\begin{array}{c}\text { Enddruck } \\
\text { in II }\end{array}$ & $\begin{array}{c}\text { pCt. H } \\
\text { aus K }\end{array}$ & $\begin{array}{c}\text { pCt. H } \\
\text { aus O }\end{array}$ \\
\hline 1 & $20^{\circ}$ & 735.6 & 465.0 & 666.0 & 699.1 & 99.61 & 99.44 \\
2 & $20^{\circ}$ & 733.6 & 465.0 & 666.6 & 700.1 & 99.82 & 99.44 \\
3 & $20^{\circ}$ & 744.1 & 470.6 & 669.1 & 700.4 & 99.88 & 99.50
\end{tabular}

Es wurden weiterhin auch Wasserstoff-Verbrennangen mit Luft statt mit annăhernd reinem Sauerstoff ausgeführt, wobei vor Zulassen der Luft bis $600 \mathrm{~mm}$ evacuirt wurde, sodass die angewendete Wasserstoffmenge $\left(B^{\prime}-600\right) \mathrm{mm}$ betrug.

\begin{tabular}{c|c|c|c|c|c|c|c}
\hline \hline No. & $t$ & $B^{\prime}$ & $p_{1}$ & $p_{2}$ & $\begin{array}{c}\text { Enddruck } \\
\text { in II }\end{array}$ & $\begin{array}{c}\text { pCt: } \mathrm{H} \\
\text { aus K }\end{array}$ & $\begin{array}{c}\text { pCt. H } \\
\text { aus O }\end{array}$ \\
\hline 1 & $20^{0}$ & 748.6 & 173.6 & 235.5 & 701.6 & 100.60 & 99.94 \\
2 & $20^{0}$ & 748.6 & 173.7 & 235.5 & 701.6 & 100.70 & 100.08
\end{tabular}

Drei nach dem zuerst beschriebenen Verfabren (Fig. 2) ausgeführte Ana-

\begin{tabular}{|c|c|c|c|c|c|c|c|c|}
\hline No. & $\begin{array}{c}\text { Dauer der } \\
\text { Verbrennung }\end{array}$ & $\left|\begin{array}{c}B^{\prime} \\
t=20^{\circ}\end{array}\right|$ & $p_{1}$ & $p_{2}$ & $\mathrm{p}_{3}$ & $\begin{array}{c}\text { Enddruck } \\
\text { in II }\end{array}$ & $\begin{array}{c}\text { pCt. } \mathbf{H} \\
\text { aus } \mathbf{K}\end{array}$ & $\begin{array}{l}\text { pCt. } \mathrm{H} \\
\text { ans } \mathrm{O}\end{array}$ \\
\hline 1 & 5 Min. & 742.1 & 518.1 & 539.5 & 681.5 & 701.3 & y9.97 & 100.05 \\
\hline 2 & $1 \gg$ & 741.1 & 509.2 & 541.0 & 679.0 & 698.5 & 99.77 & 100.8 \\
\hline 3 & 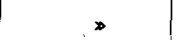 & 738.6 & 499.7 & 542.5 & 679.7 & 699.3 & 99.74 & 100.01 \\
\hline
\end{tabular}
lysen licferten folgende Ergebnisse '):

Verbrennung von reinem Kohlenoxyd.

Das zu den folgenden Versuchen dienende Gas wurde aus Oxalsäure und concentrirter Schwefelsäure entwickelt, durch aufeinanderfolgendes Waschen mit Kalkbrei und starker Kalilauge von Kohlen-

1) Angewendet: Sauerstoff von $89.8 \mathrm{pCt}$, Kolbenverhältniss $\frac{\mathbf{v}_{2}}{\mathbf{v}_{1}}=\frac{1}{1.003}$. 
säure befreit and aus einem Quecksibergasometer zur Bestimmung entnommen. Mit Rücksicht auf letzteren Umstand wurde die Füllung des Gaskolbens unter möglichst geringem Verbrauch an Gas ausgeführt und deshalb umgekehrt wie bei der Füllung mit Wasserstoff verfahren. Es wurde nämlich der Gaskolben zunächst durch Verdrängen oder durch wiederholtes Evacuiren und Einlassen mit Sauerstoff gefüllt, auf $400 \mathrm{~mm}$ Minderdruck evacuirt und dann Kohlenoxyd bis zum Gleichgewicht mit dem äusseren Druck zugelassen.

Dementsprechend beträgt hier der Partialdruck des Koblenoxyds $400 \mathrm{~mm}$, der des Sanerstoffs $\left(B^{\prime}-400\right) \mathrm{mm}$. Für dic Contraction gilt, wie bei Wasserstoff, die Formel

$$
\dot{\mathrm{K}}=\mathrm{B}^{\prime}-\frac{\mathrm{v}_{2}}{\mathrm{v}_{\mathrm{l}}}\left(\mathrm{i} 00-\mathrm{p}_{1}\right)
$$

für den Sauerstoffverbrauch wird dieselbe

$$
0=\left(B^{\prime}-400\right) a-\frac{v_{2}}{v_{1}}\left[\left(p_{3}-p_{2}\right)-\left(B^{\prime}-700\right) 0.21\right] ;
$$

es tritt also $B^{\prime}-400$ an Stelle ron 400 und die Indices von $p$ ändern sich, weil hier die Bestimmung der bei der Verbrennung entstandenen Kohlensäure $\left(p_{2}-p_{1}\right)$ der Bestimmung des Sauerstoffs $\left(p_{3}-p_{2}\right)$ vorausgeht. Die Berechnung des Kohlenoxyds aus Contraction, Kohlensäure- und Sanerstoff-Verbrauch erfolgte zunächst nach den üblichen Formeln $\left(\mathrm{O}=2 \mathrm{H}, \mathrm{CO}=\mathrm{CO}_{2}, \mathrm{CO}=2 \mathrm{O}\right)$ späterhin nach den in der vorhergehenden Abhandlung entwickelten genauen Formeln:

$$
\mathrm{CO}=1.976 \mathrm{~K}, \quad \mathrm{CO}=1.006 \mathrm{CO}_{2}, \quad \mathrm{CO}=2 \mathrm{O} .
$$

Als eine Ursache, weshalb bei der Verbrennung ron Kohlenoxyd, auch abgeseben voll der Ungenauigkeit der ursprürglichen Berechnung, zunäcbst sehr viel ungünstigere Ergebnisse erbalten wurden als bei der Verbrennung von Wasserstoff, erwies sich die erhebliche Löslichkeit der entstandenen Koblensüure im Sperrwasser. Lässt man nach der Verbrennung im Kolben II, wie es bei der Wasserstoffbestimmung obne merklichen Fehler anging, etwa $3-4 \mathrm{ccm}$ luftfreies Wasser eintreten, stellt unter Umschütteln die Temperatur des Wassermantels ein und fübrt dann die Druckbestimmung aus, so löst sich soviel Kolilensäure, dass die Bestimmung derselben nachher um $1-2 \mathrm{pCt}$. zu niedrige Werthe liefert und die Contraction entsprechend zu hoch ausfällt. Eine zweite, in derselben Richtung wirkende Feblerquelle bildet die bekannte Absorption kleiner Koblensäuremengen an feuchten Glasfächen. Der erste Fehler wurde beseitigt, als man jedes Mal nur $1 / 2 \mathrm{cem}$ evacuirtes Wasser in den Kolben eintreten liess, nachdem vorher die Temperatur eingestellt war, und dann, ohne umzuschütteln, der Kolben zur Bestimmung des Minderdrucks vom Manometer auf den Saugstutzen setzte; so kam die geringe Menge Sperrwasser nur an der kleinen Oberfäche im Hals des Kolbens mit dem kohlensäurehaltigen Gase in Berührung und konnte nicht merklich lösend wirken. Um 
Kohlensüureverlust durch Absorption an der inneren Kolbenfläche auszuschliessen, erwies es sich nothwendig, den Kolben fast trocken zu verwenden, ohne dass doch die vollständige Sättigung des Gasraumes mit Wasserdampf dadurch beeinträchtigt wurde. Damit dies mit Sicherheit erreicht würde, wurde der Kolben II vor der Bestimmung mit Alkohol und $A$ ether ausgespült und zum Trocknen unter Einfübrung einer Messingcapillare ein starker Luftstrom durchgesaugt, nachdem der Wassermantel mit heissem Wasser gefüllt worden war. Sobald die Temperatur des Wassers auf etwa $40^{\circ}$ gesunken war, wurde mittels der Capillaren ein Tröpfchen Wasser auf den Boden des Gaskolbens gebracht und dieser dann anf etwa $600 \mathrm{~mm}$ evacuirt; dabei darf der Wassertropfen nicht völlig versehwiuden. Dann wird die Versuchstemperatur eingestellt und auf $700 \mathrm{~mm}$ weiter evacuirt.

Bei Berücksichtigung dieser Vorsichtsmaassregeln und unter Benutzung der oben angeführten genauen Formeln für die Berechnung lieferte die Verbrennung von reinem Koblenoxyd mit Sauerstoff (von $98.9 \mathrm{pCt}$.) befriedigend übereinstimmende Werthe. Die Verbrennungsdauer war, wie bei den Wasserstoffbestimmungen, etwa eine Minute.

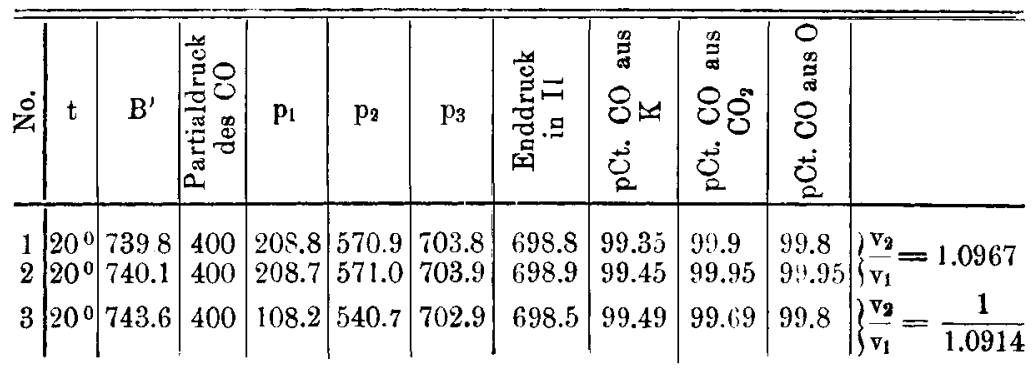

Entsprechende Bestimmungen mit dem gleichen Koblenoxyd unter Anwendung von Luft statt Sauerstoff ergaben:

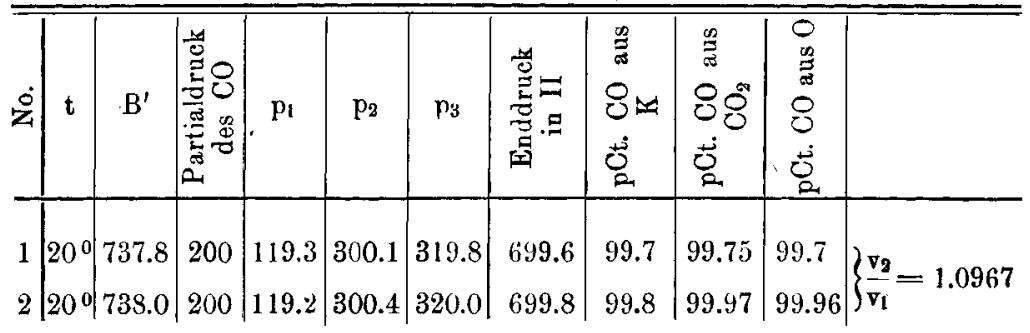

Leuchtgas - Analyse.

Die Wiederholung der mit dem Kohlenoxyd durchgeführten Versuche an reinem Metban hätte besondere Schwierigkeiten geboten, da

Berichte d. D. chem. Gesellschaft. Jahrg. XXXVII. 
alle früheren gasanalytischen Untersuchungen ${ }^{1}$ ) am Methan gezeigt haben, dass auf den bisher üblichen Wegen kein röllig reines Gas erhalten wird. Die Untersuchung erschien auch nicht nothwendig, da die Verbrennungsproducte dieselben sind wie beim Wasserstoff and Kohlenoxyd, für welche die Methode erprobt war. Die einzige Möglicbkeit einer Abweichung konnte darin liegen, dass das Methan schwerer verbreunlich ist. Das musste sich aber ebenso sicher bei der gemeinsamen Verbrennung von Wasserstoff, Koblenoxyd und Methan feststellen lassen, weil ja bei jeder Analyse in dem Enddruck, wie schon früher dargelegt, ein scharfes Kennzeichen für die Ab. wesenheit unverbrannter Bestandtheile im übergeströmten Gase geboten ist. Als nächster Schritt war demnach die gemeinsame Verbrennung der drei Gase vorzunehmen.

Ein solches Gemisch liess sich herstellen. indem aus Leuchtgas die absorbirbaren Bestandtheile entfernt wurden; das aber konnte am einfachsten mit der analytischen Bestimmung verbunden werden. Und 80 wurde zur Anwendung der vorbeschriebenen Methode anf die vollständige Analyse des Leuchtgases übergegangen.

Art und Behandlung der zu verwendenden beiden Gaskolben ist oben beschrieben; Kolben I wird mit $700 \mathrm{~mm}$ des zu untersuchenden Gases gefüllt und Kohlensäure und Sauerstoff absorptiometrisch bestimmt; die hierbei gefundenen Minderdrucke seien $p_{1}$ und $p_{3}$. Bei dem sehr geringen Sauerstoffgehalt ist die Absorption meist so träge, dass das Reagens nicht ohne weiteres eingesaugt wird. Man giebt dann entweder Eiswasser in den Kühlmantel oder man presst die Flüssigkeiten hinein. Hierzu wird der Hahnansatz mit der Flüssigkeit gefüllt, danu über das Ende desselben ein Gummischlauch gezogen und mit dem Munde in diesen Luft hineingepresst. Oeffnet man dabei den Hahn capillar, so tritt die Flüssigkeit in Folge des Ueberdrucks in den Kolben.

Absorption der schweren Kohlenwasserstoffe. Trotz der merklichen Löslichkeit von Aethylen und Benzol in wässrigen Flüssigkeiten ist es, wie die Rechnung zeigt, zulässig, die Sauerstoff bestim. mung vor der Bestimmung des sch weren Koblenwasserstoffes auszuführen, da der Partialdruck der Letzteren gering ist und die für die Sanerstoffbestimmung erforderliche Flüssigkeitsmenge nur $5 \mathrm{ccm}$ beträgt. Zunächst muss die an den Wandungen anhaftende alkalische Pyrogallollösung, die ja erhebliche Mengen Kohlensäure enthält, durch Ausspülen mit Wasser entfernt werden. Bei Minderdrucken bis ungefähr $100 \mathrm{~mm}$

1) Vergl. die von Bunsen mitgetheilten Analysen, ferner Drehschmidt, diese Berichte 21, 3250; Dennis und Hopkins, Zeitschrift für anorg. Chem. 19, 179. 
genügt lufthaltiges Wasser; darüber hinaus nimmt man zweckmăssig Wasser, das kurze Zeit bei ungefäbr demselben Minderdruck geschüttelt worden ist. Das Ausspülen geschieht jedesmal mit $2 \mathrm{ccm}$ Wasser; dasselbe wird eventuell nicht in den Saugstutzen, sondern in ein Saugfläschchen abgesaugt, sofern man zur Sicherheit die abgesaugte Flüssigkeit mit Baryumhydroxyd auf Kohlensäure prüfen will. Ein dreimaliges Ausspülen genügt völlig. Ein Fehler entsteht durch Lösen von Aethylen und Benzol nicht, weil diese ja unmittelbar danach durch Absorption bestimmt werden sollen.

Um die rauchende Schwefelsäure nicht unnütz mit Wasser zu verdünnen, spült man zuerst mit concentrirter Schwefelsäure aus, die Wasser und den Wasserdampf wegnimmt Die Schwefelsäure wird dann wieder möglichst vollständig abgesaugt und sofort $10-15 \mathrm{ccm}$ rauchende Schwefelsäure von ca. $7 \mathrm{pCt}$. Schwefelsäureanhydrid eingeführt. Ist der Gehalt an schweren Koblenwasserstoffen so gering, dass nicbt die ganze vorgeschriebene Flüssigkeitsmenge nach kurzem Schütteln eintritt, so macbt das keinen Fehler, da ja dann auch weniger Absorptionsflüssigkeit gebraucht wird. Nun wird 5 Min. gelinde geschüttelt, wieder abgesaugt und darauf mit concentrirter Schwefelsäure ausgespült Dann wird zweimal mit je $2 \mathrm{ccm}$ verdünnter Schwefelsäure (1.16 spec. Gewicht) ausgespült und darüber der Minderdruck bestimmt, weil Säure dieser Concentration dieselbe Tension hat, wie die zuvor verwendete Kalilauge von 1.16 spec. Gew. Wenn man zuerst verdünnte Schwefelsäure zur concentrirten einsaugt, darf man den Kolbenhahn nicht öffnen, bevor die beim Mischen eingetretene Temperaturerhöhung wieder ausgeglichen ist, weil sonst Gas austreten würde. Der hier gefundene Minderdruck unter Berücksichtigung der Flüssigkeitssäule im Habnansatz sei $p_{3}$.

Verbrennung des Gasrestes. Um aus dem Kolben I die Schwefelsäure zu entfernen, wird zweimal mit je $2 \mathrm{ccm}$ Wasser ausgespült; dabei verwendet man luftgesättigtes oder theilweise entluftetes Wasser je nacb dem zuletzt beobachteten Druck.

Das Gas muss jetzt soweit vermindert werden, dass die verbliebene Menge mit einer zur Verbrennung ausreichenden Menge Sauerstoff oder Luft gemischt werden kann. Nach der Absorption der schweren Kohlenwasserstoffe sind noch $B^{\prime}-p_{3}$ mm Gas im Kolben. Man muss also auf einen passenden Minderdruck $p$ evacuiren und dann durch Füllen bis zum Gleichgewicht mit dem äusseren Druck $\mathrm{p} \mathrm{mm}$ Sauerstoff oder Luft zulassen. Dann sind nachber die gefundenen mm Wasserstoff, Kohlenoxyd und Grubengas mit $\frac{B^{\prime}-p_{3}}{B^{\prime}-p}$ zu multipliciren, um die unmittelbar erbaltenen Werthe auf die ursprünglich angewendete Gasmenge (700 $\mathrm{mm}$ Partialdruck) umzurechnen. 
Es empfieblt sich, den Druck p so zu bemessen, dass die rückständige Gasmenge in einem einfachen Zahlenverhältniss, $1 / 2,1 / 3$ etc. zu der zuvor vorbandenen Gesammtmenge steht, je nachdem es für das Mengeuverhältniss des Sauerstoffs zu dem brennbaren Gase passt. Dieser Minderdruck, bis zu dem evacuirt werden muss, ergiebt sich dann z. B. für die halbe Gasmenge $z u p=\frac{B^{\prime}-p_{3}}{2}+p_{3}=\frac{B^{\prime}+p_{3}}{2}$. Man braucht so binterber die gefundenen mm für die einzeluen Gasbestandtheile nur mit $2 / 7$ zu multipliciren, um Procente zu erhalten.

Die Einstellang auf $\mathrm{p} \mathrm{mm}$ wird folgendermaassen ausgeführt. Um etwaige Diffusion von Gas aus dem Kolben in den Stutzen binein zu vermeiden, saugt man ruckweise ab, indem man nach dem Aufsetzen des Kolbens auf den Stutzen bei geschlossenem Kolbenhahn auf den einzustellenden Druck evacuirt, den Kolbenhahn einen Augenblick öffnet und wieder schliesst. Dadurch sinkt die Quecksilbersäule; man stellt wieder ein, öffnet und schliesst den Kolben wieder und so fort, bis der betreffende Minderdruck im Kolben erzengt ist. Dieses Verfahren ist sehr schnell ausführbar und schliesst jede Gasmischung durch Diffusion aus. Nun lässt man den zur Verbrennung dienenden Sauerstoff bis zum Gleichgewicht mit dem äusseren Drack mittels des oben beschriebenen Einfüllapparates (Fig. 1) zu, schliesst den Kolbenhahn und füllt den Hahnansatz mit Wasser.

Unterdessen hat man schon den zweiten Gaskolben vorbereitet, d. b. wie I getrocknet, dann mit Wassertröpfehen versehen und bei der bestimmten Temperatur auf $700 \mathrm{~mm}$ eracuirt. Um den Hahn für längere Zeit verschlusssicher zu machen, wird in den Habnansatz Wasser gegeben und durch capillares Oeffnen des Hahnes in die Durchbohrung eingefüllt. Das übrige Wasser wird dann aus dem Hahnansatz herausgeschleudert, wobei das Küken nach oben zu halten ist. Man erwärmt nun den Hahnansatz etwas in der leuchtenden Flamme eines Bunsen-Brenners und saugt, um ihn zu trocknen, einen Luftstrom durch. Jetzt wird der Kolben mittels eines kurzen Stückes Druckschlauch an die rechte Seite der Platincapillare ') (Fig. 3) angeschlossen.

1) Man prüft dieselbe auf ihre Dichtigkeit, indem man sie am Manometer evacuirt und ungefähr 1/4 Stunde so belässt. Als Druckschlauch für die bei der Platincapillare nothwenòigen Gummiverbindungen empfiehlt sich ein rother Schlauch von etwa $9 \mathrm{~mm}$ Stärke und $2 \mathrm{~mm}$ Durchbohrung. Um die Berührungsetellen von Messing and Gummi vacuamsicher zu machen, bestreicht man die Messingtheile vor Ueberzieben des Schlauches mit einer dieken Lösung von Kautschuk in Chloroform. 
Nach Ansetzen der zwei Mal rechtwinklig gebogenen Messingcapillare (Fig. 3 a) an die andere Seite wird der verbindende Druckschlauch hier mit einer kräftigen Klemmschraube geschlossen, das freie Ende des Messing-T-Stückes ${ }^{1}$ ) mit der Wasserstrablpumpe verbunden und anoähernd vollständig eracuirt.

Unterdessen setzt man ein Gefäss mit luftgesättigtem Wasser unter den linken Theil der Capillare, sodass die zwei Mal gebogene Messingeapillare mit ihrem unteren Theil möglichst tief eintaucht. Jetzt spiesst man den oben vorbereiteten Kolben I, der das zu analysirende Gas enthält, auf die Capillare; dazu öffuet man bei nach unten gehaltenem Hahnansatz den Hahn des Kolbens völlig, sodass durch den Zug des im Hahnansatz befindlichen Wassers das Gas sich bis in den Hahnansatz binein ausdehnt, wäbrend der gleiche Rauminbalt, nämlich 2-3 Tropfen. Wasser abtropfen. Man schliesst den Hahn wieder und steckt den Kolben, ohne denselben aus seiner senkrechten Lage zu entfernen, mit dem Hahnansatz auf die Capillare, öffnet den Habn wiederum und führt den Kolben hinab, sodass der Hahnansatz tief in das untergestellte Wassergefäss eintaucht, wäbrend der oberste Theil der Glaswandung dicht auf der Oeffnung der Messingcapillare aufliegt; in dieser Stellung wird der Kolben durch einen Ring oder eine Klammer festgehalten.

Inzwischen ist der todte Raum evacuirt. Man schnürt dicht am Messing-T-Stück A ab und spritzt dabei in den freiwerdenden Theil der Gummidurchbohrung Wasser, wodurch ein sicherer Verschluss erreicht wird. Nun wird der zur Erbitzung der Platincapillare dienende Fächerbrenner angezündet und nach dem Glühen derselben der Quetschhahn geöffnet, der den Zugang vom Gaskolben zur Platincapillare verschliesst. Dadurch tritt schon etwas Gas aus dem Kolben in den ericuirten Raum und rerbrennt. Jetzt kann man den Hahn des hintergelegten Kolbens II allmäblich völlig öffuen; die Schnelligkeit der Verbrennung wird an dem Nachsteigen des Wassers in den Kolben I verfolgt. Man regulirt den Hahn des Kolbens II so, dass bei einem Kolbeninhalt von ungefähr $100 \mathrm{ccm}$ die Verbrennungsdauer ${ }^{3} / 4-1 \mathrm{Min}$. beträgt.

Sobald die letzte Gasblase aus dem Kolben in die Messingcapillare tritt, entfernt man die Flamme und bläst zur Kühlung auf die glübende Stelle der Capillare. Alsdann rerlischt dieselbe, und es tritt in ihr ein scharfes, knisterndes Geräusch auf. Jetzt ist der hinter-

1) Zweckmässig wird das Messing-T-Stück ein für alle Mal mit der Platincapillare fest verbunden. 
gelegte Kolben II schräg nach oben zu halten, damit das Wasser, das nunmehr durch die erkaltete Platincapillare hindurchtritt, den Gasinhalt des Hahnansatzes vor sicb her in den Kolben II hineintreiben kann. Der Kolbenhahn ist zu sehliessen, sobald das Wasser die Hahndurchbohrung erseicht hat.

Man löst jetzt den Kolben von der Gummiverbindung und nimmt den Apparat anseinander. Die Platincapillare und die zwei Mal gebogene Messingcapillare setzt man jede für sich an eine Pumpe an und saugt Luft durch, um sie zu trocknen.

Bestimmung des Minderdrucks im KolbenII, der gebildeten Kohlensäure und des unverbrauchten Sauerstoffs. Im Kolben II selbst stellt man jetzt wieder die Temperatur ein, sangt $1 / 2 \mathrm{ccm}$ Wasser hoch und setzt den Kolben, ohne ihn aus seiner senkrechten Lage zu bringen, auf den Saugstutzen und bestimmt den Minderdruck. Derselbe se: p4. Zur Absorption der Kohlensäure verwendet man 2-3 ccm Kalilauge (1.16 spec. Gew.). Der gefundene Minderdruck sei $p_{s}$. Hier wie später hat man wegen des schon hohen Minderdrucks darauf zu achten, dass man immer etwas Flüssigkeit im Hahnansatz behält, damit nicht bei einer etwaigen Undichtigkeit des Hahnes Luftbläschen nachgesaugt werden können.

Es folgt nun als letzte Bestimmung diejenige des unverbrauchten Sauerstoffs. Hierzu benutzt man ebenfalls wegen des hoben Minderdrucks luftfreie Reagentien; man erbält diese am besten so, dass man die gerade erforderliche Menge des Absorptionsmittels unmittelbar vor den Gebrauch im Reagensglas aufkocht und unter Wasser abkühlt. Nach dem Einsaugen von $2 \mathrm{ccm}$ Pyrogallol und $3 \mathrm{ccm}$ Kalilauge (33 pCt.) wird 1 Min. geschüttelt. Unterdessen evacuirt man Wasser auf den zu erwartendem Minderdruck (etwa $600-700 \mathrm{~mm}$ ) und schüttelt krïftig durch. Man saugt daun aus dem Gaskolben, ohne erst die Tewperatur einzustellen, die alkalische Pyrogallollösung ab '), spült ein Mal mit $2 \mathrm{ccm}$ dieses evacuirten Wassers aus, saugt wiederum $2 \mathrm{ccm}$ ein und bestimmt darüber nach Einstellung der Temperatur den Minderdruck; derselbe sei $p_{6}$.

Berechnung der Analyse: Bei constanter Temperatur und dem um die $W$ assertension corrigiten Barometerstand $B^{\prime}$ werden experimentell erhalten

1) Die Ablesung direct über Pyrogallolkali empfiehlt sich bei hohen Minderdrucken nicht, weil unter diesen Umständen das abgesaugte Absorptionsmittel mit der im Saugstutzen befindlichen. Salzsāure Kohlensäure entwickelt, was zu erheblichen Druckschwankungen führen kann. 


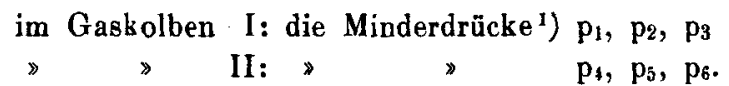

Das Volumenverhältniss der beiden Kolben ist: $\frac{\nabla_{9}}{\nabla_{1}}$; der Partialdruck der angewendeten Gasmenge: $700 \mathrm{~mm}$.

Dann ergiebt sich:

$$
\begin{gathered}
\mathrm{CO}_{2}=\mathrm{p}_{1} \mathrm{~mm} \text { oder } \frac{\mathrm{p}_{1}}{7} \mathrm{pCt} . \\
\mathrm{O}=\left(\mathrm{p}_{2}-\mathrm{p}_{1}\right)-\left(\mathrm{B}^{\prime}-700\right) \cdot 0.21 \mathrm{~mm} \\
\quad \text { oder }\left\{\frac{\left(\mathrm{p}_{2}-\mathrm{p}_{1}\right)}{7}-\left(\mathrm{B}^{\prime}-700\right) \cdot 0.03\right\} \text { pCt. }
\end{gathered}
$$

schwere Kohlenwasserstoffe: $\left(p_{3}-p_{2}\right)$ mm oder $\frac{p_{3}-p_{2}}{7} p C t$.

Für den Fall, dass die Hälfte des ursprünglich verwendeten Gases zur Verbrennung kommen kann, ergiebt sich aus $p_{3}$ der Werth für den Minderdruck, bis zu welchem der Kolben I zu evacuiren war ${ }^{2}$ ), zu $\mathrm{p}=\frac{\mathrm{B}^{\prime}+\mathrm{p}_{3}}{2}$; den gleichen Werth hat der Partialdruck des zugelassenen Sauerstoffs, dessen Gehalt $u$ sei. Dann ist, auf Kolben I und die Gesammtmenge des Gases bezogen:

Contraction: $K=\left\{B^{\prime}-\frac{v_{2}}{v_{1}}\left(700-p_{4}\right)\right\}$,

gebildete $\mathrm{CO}_{2}: \frac{\mathrm{v}_{9}}{\mathrm{v}_{1}}\left(\mathrm{p}_{5}-\mathrm{p}_{4}\right)$,

verbrauchter Sauerstoff: $O=\left\{\alpha \cdot p-\frac{v_{2}}{\nabla_{1}}\left[\left(p_{4}-p_{5}\right)-\left(B^{\prime}-700\right) \cdot 0.21\right]\right\}$.

Aus den Werthen für Contraction, entstandene Kohlensäure und verbrauchten Sauerstoff endlich erbält man nach den in der vorhergehenden Abhandlung entwickelten Gleichungen:

$$
\begin{aligned}
& \mathrm{H}_{2}=\mathrm{K}-\mathrm{O}-0.006 \mathrm{CO}_{2}, \\
& \mathrm{CO}=1 / 3 \mathrm{~K}-\mathrm{O}+4 / 3 \mathrm{CO}_{2}+0.006 \mathrm{CO}_{2}, \\
& \mathrm{CH}_{4}=-1 / 3 \mathrm{~K}+\mathrm{O}-1 / 3 \mathrm{CO}_{2}
\end{aligned}
$$

die drei rerbrennlichen Gase in Millimetern und durch Multiplication mit $2 / 7$ in Procenteu.

1) Der Minderdruck wird gemessen durch die Manometerablesung, vermohrt um den Quecksilberwerth der Fuãssigkeitsqŭule in Fahnansatz, wie früber dargelegt worden ist.

$\left.{ }^{2}\right)$ Kommt z. B. nur ein Drittel des Gases zur Verbrennung, so ergiebt sich der Minderdruck aus $p=2 \frac{\left(B^{\prime}-p_{3}\right)}{3}+p_{3} z a \frac{2 B^{\prime}+p_{3}}{3}$ etc. 
Der vorhandene Stickstoff ergiebt sich dann aus:

$$
\mathrm{N}=\mathrm{B}^{\prime}-\left(\mathrm{H}_{2}+\mathrm{CO}+\mathrm{CH}_{4}\right)-\mathrm{p}_{3}-\left(\mathrm{B}^{\prime}-700\right) \cdot 0.79
$$

in Millimetern und durch Division mit 7 in Procenten.

Im Kolben II muss sich unter Hinzarechnung der vöthigen Werthe wieder der Enddruck 700 einstellen. Derselbe berechnet sich aus

$$
E=p_{6}-\left(B^{\prime}-700\right) \cdot 0.21+\frac{v_{2}}{v_{1}}\left[\frac{\left(B^{\prime}-700 \times 0.79+N\right.}{B^{\prime}-p_{3}} \cdot\left(B^{\prime}-p\right)+(1-\alpha) p\right] .
$$

\section{Beleganalysen.}

Die nach dieser Vorschrift ausgeführten Leuchtgasanalysen zerfallen iu 2 Gruppen; bei der ersten warde noch der Partialdruck des zur Verbrennung nöthigen Sauerstoffs ungefähr passend gewählt, bei der zweiten Gruppe wurde derselbe so berechnet, dass die Hälfte des ursprünglichen Gases zur Analyse kam. Bei der Gruppe 1 war der verwendetete Sauerstoff 98.9-procentig; bei der Gruppe 2 wurde 89.8procentiger Saner=toff benuzt. Bei allen Analysen ist $\frac{\mathrm{v}_{2}}{\mathrm{v}_{1}}=1.0967$.

Das Leuchtgas der 2. Gruppe ist ein anderes, als das der 1. Gruppe. Die Dauer einer vollständigen Analyse betrug $2-2 \frac{1}{2}$ Stunde.

\begin{tabular}{|c|c|c|c|c|c|c|}
\hline & I & II & III & IV & V & VI \\
\hline $\mathrm{t}$ & $20^{\circ}$ & $20^{\circ}$ & $20^{\circ}$ & $20^{\circ}$ & $20^{n}$ & $20^{\circ}$ \\
\hline $\mathbf{B}^{\prime}$ & 7466 & 733.7 & 742.6 & 738.6 & $7+2.1$ & 7434 \\
\hline$p_{\mathrm{i}}$ & 10.5 & $1 \% .1$ & 11.1 & 11.9 & 11.1 & 11.9 \\
\hline$p_{2}$ & 23.9 & 24.5 & 24.0 & 23.1 & 23.4 & 24.1 \\
\hline$p_{3}$ & 44.6 & 451 & 45.4 & 44.3 & 44.1 & 45.4 \\
\hline$p$ & 500.0 & 400.0 & 400.0 & 4000 & 400.0 & 400.0 \\
\hline$p_{4}$ & 337.9 & 465.7 & 469.6 & 471.0 & 470.1 & 469.3 \\
\hline$p_{5}$ & 4201 & 578.9 & $581 i .1$ & 586.2 & 586.7 & 585.4 \\
\hline & 6835 & 676.6 & 679.5 & 680.5 & 679.7 & 678.7 \\
\hline Enddruck in II & 6998 & 699.7 & 699.6 & 699.6 & 699.6 & 699.6 \\
\hline $\mathbf{K}$ & 349.5 & 478.7 & 489.9 & 487.8 & 490.0 & 490.4 \\
\hline $\mathrm{CO}_{2}$ & 90.2 & 124.2 & 127.8 & 126.4 & 127.9 & 127.4 \\
\hline verbr. 0 & 216.3 & 296.6 & 303.1 & 300.9 & 303.4 & 303.3 \\
\hline Gehalt an $\mathrm{CO}_{2}$ & 1.54 & 1.73 & 1.59 & 1.70 & 1.59 & 1.70 \\
\hline$\Rightarrow \quad \geqslant 0$ & 0.47 & 0.7 & 0.57 & 0.44 & 0.5 & 0.44 \\
\hline $\begin{array}{c}\bullet \text { schw. } \\
\text { Kohlenwasserst. }\end{array}$ & 3.10 & 2.94 & 3.06 & 3.03 & 2.96 & 3.04 \\
\hline Gebalt an $H$ & 53.97 & 53.34 & 54.06 & 54.51 & 54.17 & 54.13 \\
\hline$\gg \mathrm{CO}$ & 8.58 & 8.57 & 8.93 & $9.0 j$ & 9.10 & 9.03 \\
\hline - $\mathrm{CH}_{4}$ & 28.34 & 28.07 & 28.40 & 28.15 & 28.43 & 28.23 \\
\hline$\Rightarrow \quad \geqslant N$ & 4.10 & 4.65 & 3.35 & 3.13 & 3.24 & 3.43 \\
\hline Summe der pCt. & 100.10 & 100.00 & 100.01 & 100.01 & 99.99 & 100.00 \\
\hline
\end{tabular}

Gruppe 1. 
Gruppe II.

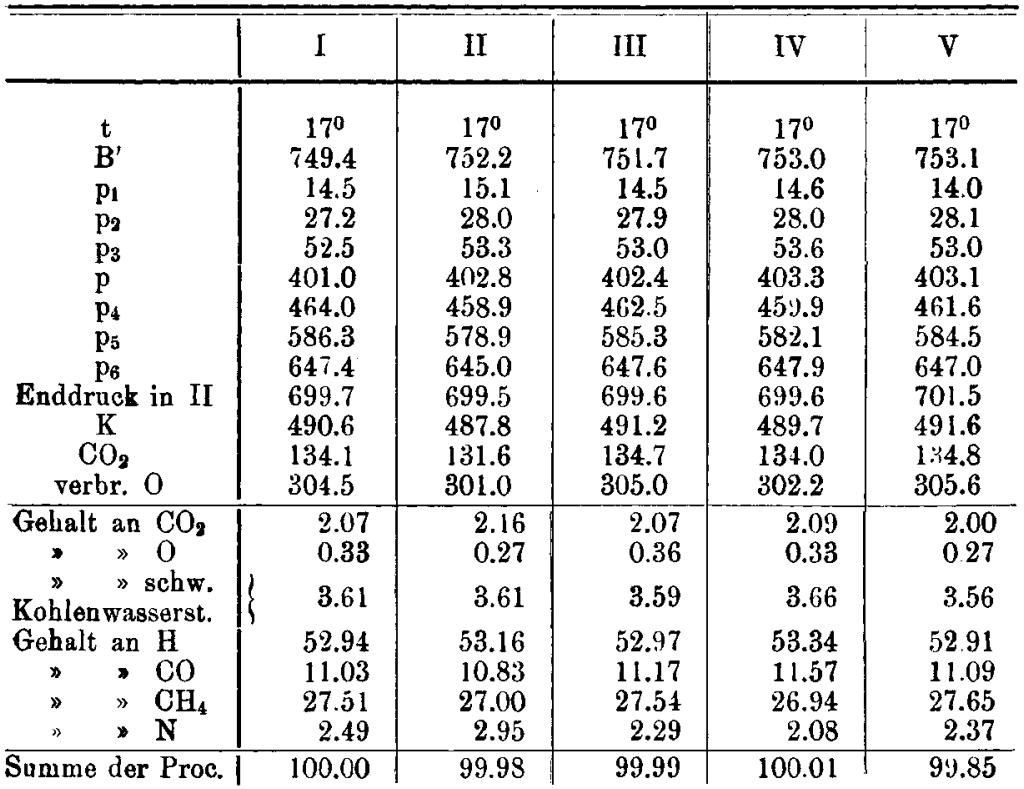

71. A. Wohl: Bestimmung des Metallgehaltes im Zinkstaub. (8. Mittheilung über gasometrische Bestimmungen in Gaskolben.)

[Aus dem I. Berl. Universitäts-Laboratorium.]

(Eingegangen am 28. Januar 1904.)

Durch eine eingehende Untersuchung ron de Koninck') und Grandry ist neuerdings festgestellt worden, dass von den Methoden zur Bestimmung des Metallgehaltes im Zinkstaub das Verfahren von Fresenius, Messung des durch Salzsäure und Platinchlorid entwickelten Wasserstoffes, die zuverlässigsten Ergebnisse liefert, sofern bei der Ueberleitung des Wasserstoffes in das Messrohr jede Kautschukleitung vermieden wird. Die Autoren fanden mit reinstem $\mathrm{Zink}$ (in Spähnen) 99.70 und 99.77 pCt. Metall und ziehen für die Zinkstaubanalyse diesen Werth als Reactionsgrenze in Rechnung; die Beschreibung des benutzten Apparates ist vor kurzem von de Kouinck ${ }^{2}$ ) gegeben worden.

1) Bull. de l'assoc. Belge d. chim. 16, 28t [1902].

2) Bull. de l'assoc. Belge d. chim. 17, 112 [1903]. 